\title{
Corticosteroids in sports-related injuries: Friend or Foe
}

\author{
A Rotunno, DC Janse van Rensburg, ${ }^{*}$ CC Grant, A Jansen van Rensburg \\ Institute of Sport, Exercise Medicine \& Lifestyle Research, and Section Sports Medicine, University of Pretoria, South Africa
}

*Corresponding author, email: christa.jansevanrensburg@up.ac.za

\begin{abstract}
Corticosteroids act as potent anti-inflammatory drugs and have been used in various sport settings for the treatment of both acute and chronic injuries. Basic physiology and mechanisms of action for gluco- and mineralocorticoids are discussed. Methods of administration, the action on the inflammatory response, and potential short and long-term side effects of corticosteroid use are also deliberated. More specifically, corticosteroid use in the treatment and management of sport-related injuries are discussed, elucidating on the ethical boundaries and anti-doping regulations pertaining to corticosteroid use in sports, as well as putting forth suggestions for the use of local steroid injections and their contraindications. In conclusion, it was found that, despite some controversy in the use of corticosteroid treatment in the sports environment, little empirical evidence exists that could conclusively rule for or against its use. It is however clear that, if clinically justified, there is a significant role for corticosteroid treatment in the realm of sports injury and notably with a relatively low risk profile, if administered correctly.
\end{abstract}

Keywords: Steroid hormones, athlete, sports, injury, inflammation.

\section{Introduction}

Corticosteroids are used in the sports arena in many different forms and for many different reasons. They are potent antiinflammatory drugs that inhibit the inflammatory cascade, and are thus seen as useful adjuncts in the treatment of some sportsrelated injuries. On the basis of their ability to down regulate the immune response, corticosteroids have been used extensively in the management of sports injuries to promote rapid return to the field of play. But to what extent do they affect soft tissues, the athlete, and overall performance? In line with current literature, the following exposition will attempt to explain the basic concept of corticosteroids, routes of administration, their side effects, relevance and clinical application in sports injuries, as well as the ever-present potential for abuse and current use in sports doping and performance enhancement.

\section{Corticosteroids in general}

Corticosteroids are a class of steroid compounds comprising of two main physiological groups, namely the glucocorticoids and the mineralocorticoids. However, the term "corticosteroids" is generally used to refer to glucocorticoids. They are four-ringed steroid hormones with their common biochemical precursor being cholesterol. ' Glucocorticoids are closely related to cortisol, which is an endogenous hormone produced in the cortex of the adrenal gland.
Specifically, glucocorticoids (e.g. cortisol) have multiple roles. They are important in the breakdown of proteins to amino acids, serve as an insulin antagonist by inhibiting cellular glucose uptake and breakdown, suppress immune function, and are supportive of other hormones like glucagon and growth hormone in the gluconeogenic pathway.

Mineralocorticoids on the other hand (e.g. aldosterone) influence electrolyte (sodium and potassium) and therefore intravascular volume, blood pressure and water levels in the body. ${ }^{2}$ Due to the fact that glucocorticoid and mineralocorticoid compounds are chemically alike, there are clinically important areas of overlap seen in the effects of corticosteroids among the classes, i.e. some glucocorticoids will express mineralocorticoid effects and vice versa. This is particularly important for the prescribing clinician to remember, as they may affect physiological processes and performance at several different levels. ${ }^{3}$

There are numerous well-recognised side effects in the longterm use of corticosteroids, most of which involve suppression of the hypothalamic-pituitary axis. Some of these include avascular necrosis, osteoporosis, growth suppression, peptic ulcers, weight gain, and immune suppression. There is scant evidence on the side effect profile of short-term corticosteroid use (seven days or less). ${ }^{4}$ Another established side effect of corticosteroids is allergy and hypersensitivity. The worldwide incidence ranges from $0.5-5 \% .{ }^{5-7}$ They have therefore been classified into different 
groups according to their allergenicity and chemical structure. ${ }^{8}$ This is known as the "Coopman classification":

Group A: Hydrocortisone type, e.g. hydrocortisone, prednisolone, and prednisone

Group B: Triamcinolone acetonide type, e.g. budesonide

Group C: Betamethasone type, e.g. betamethasone, dexamethasone

Group D: Hydrocortisone-17-butyrate type, e.g. betamethasone valerate.

Due to chemical and structural similarities within each group, allergy to one drug in a particular class may indicate intolerance to other drugs in the same class. In other words, cross reaction between members of the same group can occur, but equally important is that there is not much cross reaction between the different groups. ${ }^{8}$ This is why the classification system is important for the prescribing clinician to be aware of, as a patient intolerant of one group may be sensitive to another and would therefore still have drug treatment options available to them. Due to potential corticosteroid intolerance, some patients may need to undergo allergy patch testing before any specific group can be confidently administered. ${ }^{8}$

Corticosteroids have been used in the treatment of musculoskeletal disorders for decades, with varying interindividual success and efficacy. They are potent antiinflammatory drugs, with their use being controversial due to potential side effects and influence on tissue healing. ${ }^{3}$

\section{Routes of administration include:}

- Topical: use on skin, eyes and mucous membranes

- Inhaled: use on the nasal mucosa, sinuses, bronchi, and lungs

- Local injection: Intra-dermal, intra-muscular (IM), paratendinous, intra-tendinous, periarticular, and intra-articular

- Systemic: oral, intravenously (IV) and per rectum.

\section{Understanding inflammation}

In order to understand the mechanism of action of corticosteroids in the treatment of athletic injuries, one needs a basic understanding of the inflammatory response when injury occurs in the sporting context. Furthermore, it is clinically important to differentiate soft tissue injury versus other soft tissue pathologies and arthropathies. ${ }^{9}$ It has been established that the efficacy of anti-inflammatory medications such as corticosteroids is based primarily on pathological states of diseases such as rheumatoid arthritis (RA) and osteoarthritis $(\mathrm{OA})^{10}$; however, this type of treatment has not necessarily been directly applicable to sports related injuries. In disease conditions like RA and $O A$, the primary pathology is inflammation, whereas in sports injuries, the inflammation is typically secondary to the primary structural trauma.

The process of inflammation is defined as the response of vascular tissue to physiological damage'. ${ }^{9}$ With acute sports injuries and other pathological conditions (OA, RA, auto-immune diseases), the process of inflammation is at its core. Damaged cells release antigen-like substances that stimulate an immune response, which in turn causes leukocyte proliferation. Blood flow in the region increases to transport polymorphonuclear lymphocytes, macrophages and plasma proteins to the injured area. A redistribution of arteriolar flow produces stasis and local hypoxia, resulting in infiltration of tissues by leukocytes, plasma proteins and fluid into the interstitial and extracellular compartments. This process results in the classic redness, swelling, warmth, and pain that is characteristic of the inflammatory process. ${ }^{3,9}$ Nonetheless, inflammation serves several important functions. Leukocyte influx facilitates phagocytosis and removal of damaged cells and particulate matter, thus encouraging healing. The resultant pain and swelling also remind the patient to protect the affected area. However, after some time, the inflammatory response becomes counter-productive to the healing process. The extravascular pressure caused by oedema begins to slow blood flow to and from the affected area, thus delaying healing. Debris build up occurs, resulting in ongoing swelling, scar and trigger point formation, and prevention of return of normal function. ${ }^{3,9}$

Corticosteroids are one of the most common treatment modalities for chronic tendon lesions. ${ }^{11}$ There is evidence that corticosteroids are beneficial in the short-term for some tendinopathies, but conversely the intermediate and long-term effects have been shown to be less promising. ${ }^{12}$ Regarding the classification of sports injuries, there are two major groups, namely acute and chronic/overuse injuries. ${ }^{3}$ Acute injuries include macro-trauma resulting in single or multiple incidences of high force trauma to the affected soft tissue or bone, e.g. fractures, dislocations, and muscle and tendon ruptures. In these cases, inflammation is the immediate response occurring in the tissues as seen in the case of a tendinitis, which is defined as inflammation of the tendon when the musculo-tendinous junction is acutely overloaded with tensile forces that are too heavy or too sudden. ${ }^{13}$ On the other hand, chronic injuries involve multiple insults of micro-trauma, which results from chronic repetitive stress to local tissues, for example with a tendinosis, which is defined as the continual degeneration of tendon collagen due to overuse, and hence a predisposition to injury. The most important goal of a corticosteroid is to reduce inflammation. The clinician must be aware of the difference between a tendinosis and a tendinitis because of the differing treatment goals and timelines. ${ }^{13}$ Inflammation is only present in tendinitis, and generally not in tendinosis. ${ }^{14}$ Treatment with corticosteroids is generally warranted in inflammatory conditions only.

The side effect profile of corticosteroids is of clinical relevance to both the clinician and the athlete, and benefits versus risks need to be carefully weighed up..$^{15}$ Due to the wide range of adverse effects, it is imperative that the patient is fully informed of the potential risks, and reassured that the treating physician is knowledgeable of the applicability of the drugs to a particular injury and patient. The general (systemic) side effects are sometimes referred to as Cushingoid symptoms due to their presence in Cushing's disease. Short-term and long-term side effects are summarised in Table I. ${ }^{16}$ 
Table I: Systemic side effects of short-term and long-term corticosteroid use

\begin{tabular}{ll}
\hline Short-term use & Long-term use \\
\hline Cutaneous effects & Reduced bone density/osteopaenia \\
\hline Electrolyte abnormalities & Hyperglycemia \\
\hline Hypertension & Hyperlipidemia \\
\hline Hyperglycemia & Anovulation (cessation of ovulation and menstrual irregularities) \\
\hline Pancreatitis & Immunosuppression \\
\hline Haematological, immunological, and neuro-psychological effects & Adrenal insufficiency \\
\hline Fatigue & Muscle and tendon breakdown \\
\hline Restlessness/agitation & Aseptic joint necrosis \\
\hline Sleeping disturbances & Gastrointestinal, hepatic, and ophthalmologic effects \\
\hline Decreased sperm count & Growth suppression and congenital malformations are possible \\
\hline
\end{tabular}

To elaborate on the short-term side effects, reversible cutaneous effects typically include truncal obesity, acne, hyperpigmentation, petechiae, and hirsutism. Striae may also occur (irreversible). It is thought that the decreased productivity of hydroxylproline is the reason for such diminished structural stability of connective tissue. ${ }^{17,18}$ Electrolyte abnormalities mainly involve the development of hypokalemia due to mineralocorticoid effects of prednisone, prednisolone and hydrocortisone. There exists the possibility, although rare, of occurrence of electrolyte abnormalities with topical (per rectum) use of corticosteroids. ${ }^{19}$ Concerning immunological side effects, an increase in neutrophils in the circulating pool (shift caused by corticosteroid use) results in an increase of total peripheral leukocytes (14 000-20 000/(mm ${ }^{3}$ ), which will typically return to normal 1 week after steroid discontinuation..$^{20}$ Increased erythropoiesis, thrombocytosis, eosinophilopenia and monocytopenia may also develop. ${ }^{21,22}$ Taking neuropsychological effects into consideration, some symptoms reported include persistent headaches, visual symptoms, benign intracranial hypertension and seizures..$^{23,24} \mathrm{~A}$ decrease in seizure threshold is thought to occur due to electrolyte abnormalities and fluid shifts in the brain.

With regard to long-term side effects of corticosteroid use, inconclusive findings have shown some correlations with the possibility of developing or aggravating existing peptic ulcers. ${ }^{25,26,27}$ Hepatic steatosis, potentially due to hyperglycemia, may also develop, while the risk for glaucoma through intraocular pressure increases irrespective of method of administration. ${ }^{16}$ Increased intraocular pressure has been reported in pediatric populations, ${ }^{28}$ patients over the age of 40 and those with a history of glaucoma. ${ }^{29}$ Sub-capsular cataract formation has been strongly associated with prednisolone administration and may be related to covalent binding between glucocorticoid and the protein matrices of the lens, resulting in its destabilisation. ${ }^{30,31}$ Nitrogen retention, bone formation and collagen formations might also be affected by corticosteroids which even at moderate doses inhibit growth hormone release. ${ }^{16}$ In general, corticosteroid use during pregnancy is considered to be safe, ${ }^{32}$ however reports of increased risk for cleft lip and cleft palate have been put forth. ${ }^{33,34}$

\section{Corticosteroids in the sporting context}

There have been numerous studies on the applicability, efficacy, side effect profiles, and general use of corticosteroids in the management of athletes and sports injuries. ${ }^{35}$ They have been applied to a wide variety of sports-related injuries in many different contexts, some of which have been beneficial, others detrimental, to the athlete. ${ }^{12}$ Many different study designs, using human and animal subjects, examining different clinical conditions' response to local corticosteroid injections, have been performed. For the purpose of this paper, the most commonly studied conditions (tendinopathies and muscle strains) will be discussed in relation to current literature.

There is some data on the patterns of oral corticosteroid prescription, with one study finding that approximately one third of sports physicians prescribed them regularly, and that the athletes clinically benefitted from their use, and with few side effects. The authors did, however, state that repeat administration of oral corticosteroids in the same patient may pose a higher risk for medical complications..$^{35}$ Inhaled corticosteroids are generally used for exercise-induced bronchospasm or clinically proven asthma. A therapeutic use exemption in sport is not required for inhaled glucocorticoids. ${ }^{36}$ There are few good quality studies done on the effects of IM corticosteroids. Despite having no control group, one such observational study, ${ }^{37}$ found that IM corticosteroids expedite a player's return to play and reduce the game and practice times missed. Another study involving the examination of corticosteroid effects on acute iliotibial band friction syndrome demonstrated that they had improved symptoms after two weeks, but the question of whether corticosteroid injections benefit the resolution of symptoms in the long run was not answered, and therefore necessitates further research. ${ }^{38}$

The most common route of administration of corticosteroids in sports injuries is by local injection. It allows for maximisation of drug concentration at the injury site, whilst minimising the risk of systemic side effects. ${ }^{3}$

Some of the more common sports injuries treated with corticosteroid injections include: ${ }^{11,15}$ 
- Shoulder: sub-acromial bursitis; coracoid bursitis, acromioclavicular sternoclavicular joint pathology, scapulothoracic articulation, glenohumeral joint injury

- Elbow: golfer's elbow (medial epicondylitis), tennis elbow (lateral epicondylitis), olecranon bursitis

-Wrist: carpal tunnel syndrome, De Quervain's tenosynovitis

- Hand: trigger finger; triangular fibrocartilage ligament

- Knee: pes anserine bursitis, pre-patellar bursitis, iliotibial band syndrome, medial patellar plica, intra-articular tibial and fibular collateral ligaments

- Lower limb: Achilles paratenonitis, Achilles enthesitis, sinus tarsi syndrome, plantar fasciitis, inter-digital neuroma, metatarsal phalangeal injuries, retrocalcaneal bursitis, ankle sprains, posterior impingement syndrome, turf toe, Morton's neuroma.

It is important to note some of the more frequently reported local injection side effects occurring in the above-mentioned conditions: ${ }^{12}$

- Post injection pain

- Atrophy of skin

- Depigmentation of skin

- Tendon rupture

- Tendinopathy

- Septic arthritis/sepsis.

Septic arthritis has proven to be a relatively rare occurrence, providing that the technique of intra-articular injection is performed under sterile conditions. ${ }^{39}$ However, the available studies on post corticosteroid injection septic arthritis are limited both in number and scope.

The duration of corticosteroid effects have been shown to be indirectly proportional to its solubility, and low solubility drugs are hence usually favoured for injections. ${ }^{40,41}$ This does, however, predispose one to more local side effects (e.g. soft tissue and skin changes), as the drug lingers in those areas for longer. Few studies have been done on the differing preparations, dosages, and number of repeated administrations in relation to its clinical effect. A review of local corticosteroid injections indicated that a more rapid relief of symptoms was achieved with lower doses of intermediate acting drugs than with higher doses of shorter acting ones. ${ }^{42}$ Another study showed that after IM corticosteroid injection, tensile strength of the tendon improved, but repeated administration progressively weakened the same tendon, thus illustrating a relationship between cumulative dose and side effects. ${ }^{42}$

Injection into a tendon may weaken its structure, and thus increase probability of rupture. This is one of the most significant concerns related to the use of these drugs, particularly as it would adversely affect athlete performance. Studies performed in vitro on cultured human tenocytes demonstrated that corticosteroids suppress proteoglycan production by tenocytes, thereby influencing the viscoelastic properties of the tendon and increasing the potential for spontaneous rupture. ${ }^{43}$ However, in a 2010 review of randomised controlled trials ${ }^{12}$ it was noted that numerous animal studies could not confirm that corticosteroid injections caused tendon damage, and that the frequency of adverse events such as tendon rupture were generally very low. This review went on to state that more rigorous reporting of such side effects is needed. Less concerning side effects such as postinjection pain, subcutaneous atrophy and skin depigmentation were relatively common. ${ }^{12}$

Tendinopathies include a broad spectrum of conditions, often difficult to diagnose, and with considerable overlap with both tendinosis and tendinitis. A tendinopathy is not associated with inflammatory cells, and local corticosteroid therapy is not as popular as it was previously for the treatment of such lesions. ${ }^{12}$ There are few studies done on Achilles paratenonitis, demonstrating no benefit in the short-term regarding pain and return to play following corticosteroid injection. ${ }^{11,12}$ With regard to tennis elbow (lateral epicondylitis), there is strong evidence that short-term pain relief may improve, but at long-term followup there is no difference in outcome between treatment and control groups. ${ }^{11,12}$ Rotator cuff injury response to corticosteroid treatment has been conflicting. Some studies noted large shortterm effects on range of motion but not pain, whilst others noted medium-term effects in pain reduction and one study noted good long-term outcomes in overall improvement. ${ }^{12}$

Table Il: Key concepts on steroid injections ${ }^{11}$

\section{Guidelines for use of local steroid injections in tendinopathies}

- Do not use in chronic injuries unless all other intensive approaches have failed

- Useful if rehabilitation is inhibited by symptoms

- Informed consent should be obtained from the patient

- The practitioner must have full knowledge of the local anatomy

- Aseptic technique

- Use short or medium acting corticosteroid preparations, usually with added local anaesthetic

- Injection should be peritendinous, and avoid injection directly into tendons

- Minimum interval between injections should be 6 weeks

- A maximum of three injections at one site

- Soluble preparations may be useful in those patients who have had hypersensitivity/local reaction to previous injection

- Details of the injection should be carefully recorded

- Do not repeat if two injections do not provide at least 4 weeks' relief

\section{Post-injection advice}

- Patient must be advised on early post injection pain, and to avoid initial overuse

- Rest for at least 3 days to maximum 2 weeks after treatment and avoid heavy loading for 6 weeks

- Patient must not hesitate to contact doctor if any adverse reactions occur

\section{Contra-indications to corticosteroid injections}

- When pain relief and anti-inflammatory effects can be achieved by other methods

- Local or systemic infection

- Coagulopathy

- Already torn or damaged soft tissue/tendons

- Young patients 
In general, effectiveness in the longer term is unknown, and there is insufficient data on which to confidently base practice recommendations. ${ }^{12}$ Trigger finger and De Quervain's tenosynovitis ( $80 \%$ of cases) have been shown to respond well to corticosteroid treatment. ${ }^{12,44,45}$ Various other soft tissue injuries are amenable to corticosteroid treatment, however their use and effect on overall outcome is not without controversy. The treating clinician must be knowledgeable on the injury type, side effect profile and risk versus benefit ratio for their patients. Key concepts on local steroid injections for tendinopathy, as summarised by Speed (2001), are presented in Table II.

\section{Anti-doping}

Different rules apply to in-competition and out-of-competition situations. This may change from year to year and therefore needs to be validated on the World Anti-Doping Agency (WADA) list regularly.

In-competition: The systemic use of glucocorticoids is prohibited, unless a valid therapeutic use exemption (TUE) has been approved. This includes oral intake, IV or IM injection, or rectal routes. Inhalation of glucocorticoids (e.g. for asthma) as well as topical use (but be aware of hemorrhoidal creams) and injections around tendons, into joints and epidural spaces are permitted.

Out-of-competition: Topical use of glucocorticoids (e.g., anti-rash cream, hemorrhoidal creams, mucous membrane creams) is permitted. Athletes may take oral, rectal, IV or IM corticosteroids without submitting a therapeutic TUE form, providing the prohibited substance has cleared their system prior to the time defined as "in-competition". If athletes need to use these routes directly before or during competition, it is essential that they obtain TUE approval. ${ }^{36}$ Despite the fact that no evidence exists that corticosteroids are performance enhancing, oral corticosteroids are banned by WADA and the International Olympic Committee based on evidence that they were being used in large quantities by cyclists for their presumed stimulant qualities. ${ }^{36}$ Evidence of performance enhancement is only one of the three criteria for listing a banned substance. Seeing as though there is no evidence of performance enhancement of corticosteroids, the presence of the other two criteria, "harmful to health" and "against the spirit of sport", is sufficient for listing corticosteroids as a banned substance. ${ }^{4}$

\section{Conclusion}

Corticosteroid treatment of sports injuries is not without controversy. The multitude of research done on side effect profiles and effects on overall injury outcome suggests that they occupy a significant role in the treatment of patients and athletes with various pathologies. As it currently stands, too many conclusions in the literature are based on poor scientific evidence, and should not be a reason to gratuitously over-apply or abandon their use. Corticosteroids represent an additional treatment in the overall management of sports injuries, with appropriate rehabilitation and physiotherapeutic approaches being considered as the treatment of choice before drug application. Basic treatment includes 'active' rest and progressive rehabilitation within the limits of pain. With proper indications, there are only few and trivial complications that may occur with corticosteroid injections. The risks and benefits of corticosteroids must be weighed up and discussed with the athlete, together with compulsory informed consent. Athletes and clinicians also have the responsibility of understanding the anti-doping policies regarding their use.

\section{References}

1. Scott-Hickman M. Corticosteroids. In: Yamamoto LG, Inaba AS, Okamoto JK, Patrinos ME, Yamashiroya VK, editors. Case based pediatrics for medical students and residents. Honolulu: Department of Pediatrics, University of Hawaii John A. Burns School of Medicine 2004. p. 148-151.

2. McArdle WD, Katch Fl, Katch VL. Exercise physiology. Nutrition, energy and human performance. Baltimore: Wolters Kluwer Health; 2015.

3. Brukner $P$, Khan K. Treatments used for musculoskeletal conditions: more choices and more evidence. Clinical Sports Medicine $4^{\text {th }}$ ed. Sydney, Mcgraw Hill, 2013. p. 15, 174-176.

4. Brukner P, Nicol A. Use of Oral Corticosteroids in Sports Medicine. Current Sports Medicine Reports 2004;3:181-183.

5. Dooms-Goossens A, Andersen KE, Brandao FM. Corticosteroid contact allergy: an EECDRG multicentre study. Contact Dermatitis 1996;35:40-44.

6. Matura M. Corticosteroid contact allergy in Hungary. Contact Dermatitis 1998;38:225-226.

7. Khoo BP, Leow YH, Ng SK, Goh CL. Corticosteroid contact hypersensitivity screening in Singapore. Am J Contact Dermatitis 1998;9:8-91.

8. Coopman S, Degreef H, Dooms-Goossens A. Identification of cross-reaction patterns in allergic contact dermatitis from topical corticosteroids. Br J Dermatol 1989;121:2-34.

9. Leadbetter WB. An introduction to sports-induced soft tissue inflammation.. In: Mangus BC, Miller MG, Editors. Pharmacology Application in Athletic Training Philadelphia: FA Davis Company; 2005. p32-52.

10. Leadbetter WB. Anti-inflammatory therapy in sports injury. The role of nonsteroidal drugs and corticosteroid injection. Clin Sports Med. 1995 Apr;14(2):353-410.

11. Speed CA. Corticosteroid injections in tendon lesions. BMJ. 2001 Aug 18;323(7309):382-386.

12. Coombes BK, Bisset L, Vicenzino, B. Efficacy and safety of corticosteroid injections and other injections for management of tendinopathy: a systematic review of randomized controlled trials Lancet 2010;376:1751-67.

13. Bass E. Tendinopathy: Why the Difference Between Tendinitis and Tendinosis Matters. Int J Ther Massage Bodywork 2012;5(1):14-17.

14. Khan KM, Cook JL, Kannus P, Maffulli N, Bonar SF. Time to abandon the "tendinitis" myth: Painful, overuse tendon conditions have a non-inflammatory pathology [editorial] BMJ. http://www.bmj.com/content/324/7338/626.full. Published March 16, 2002. [Accessed 16 September 2011].

15. Dietzel DP, Hedlund EC. Injections and return to play. Curr Sports Med Rep 2004;3:310-315.

16. Buchman, AL. Side effects of corticosteroid therapy. J Clin Gastroenterol 2001;33(4):289-294.

17. Stratakis CA, Mastorakos G, Mitsiades NS, Mitsiades CS, Chrousos GP. Skin manifestations of Cushing disease in children and adolescents before and after the resolution of hypercortisolemia. Pediatr Derm1998;4:253-8.

18. Uitto TH, Mustakillo KK. Corticosteroid-induced inhibition of the biosynthesis of human skin collagen. Biochem Pharmacol 1972;21:2161-7.

19. Powell JR. Steroid and hypokalemic myopathy after corticosteroids for ulcerative colitis. Systemic and topical application. Am J Gastroenterol 1969;52:425-32.

20. Dale DC, Fauci AS, Wolff SM. Alternate-day prednisone: leukocyte kinetics and susceptibility to infections. N Engl J Med 1974;291:1154-8. 
21. Barr RD, Volaric Z, Koekebakker M. Stimulation of human eosinophilopiesis by hydrocortisone in vitro. Acta Haematol 1987;77:20-4.

22. King DJ, Brunton J, Barr RD. The influences of corticosteroids on human erythropoiesis. An in vivo study. Am J Pediatr Hematol Oncol 1988;10:313-5.

23. Newton $M$, Cooper BT. Benign intracranial hypertension during prednisolone treatment of inflammatory bowel disease. Gut 1994;35:423-5.

24. Levine AM, Pickett LK, Touloukian RJ. Steroids, hypertension, and fluid retention in the genesis of postoperative seizures with inflammatory bowel diseases in childhood. J Pediatr Surg 1974;9:715-24.

25. Conn HO, Poynard T. Corticosteroids and peptic ulcer: meta-analysis of adverse events during steroid therapy. J Intern Med 1994;236:619-32.

26. Messer J, Reitman D, Sacks HS, Smith H Jr, Chalmers TC. Association of adrenocorticosteroid therapy and peptic ulcer. N Engl J Med 1976;294:473-9.

27. Pecora PG, Kaplan B. Corticosteroids and ulcers: is there an association? Ann Pharmacother 1996;30:870-2.

28. Tripathi RC, Kirschner BS, Kipp M, Tripathi BJ, Slotwiner D, Borisuth NSC, et al. Corticosteroid treatment for inflammatory bowel disease in pediatric patients increases intraocular pressure. Gastroenterology 1992;102:1957-61.

29. Tripathi RC, Parapuram SK, Tripathi BJ, Y Zhong, KV Chalam. Corticosteroids and glaucoma risk. Drugs Aging 1999;15:439-50.

30. Shiono H, Oonishi M, Yamaguchi M, Sakamoto F, Umetso A, Kadowaki J, et al. Posterior subcapsular cataracts associated with long-term oral corticosteroid therapy. Ophthalmologic observations indicate these are frequent though clinically unsuspected. Clin Pediatr (Phila) 1977;16:726-8.

31. Dickerson JE Jr, Dotzel E, Clark AF. Steroid-induced cataract: new perspective from in vitro and lens culture studies. Exp Eye Res 1997;65:507-16.

32. Czeizel AE, Rockenbauer M. Population-based case-control study of tertogenic potential of corticosteroids. Teratology 1997;56:335-40.

33. Fraser FC, Sajoo A. Teratogenic potential of corticosteroids in humans. Teratology 1995;51:45-6.
34. Carmichael SL, Shaw GM. Maternal corticosteroid use and risk of selected congenital anomalies. Am J Med Genet 1999;86:242-4.

35. Madanagopal SG, Kovaleski JE, Pearsall AW. Survey of short-term oral corticosteroid administration by orthopaedic physicians in college and high school athletes. J Sports Sci Med 2009 Mar;8(1):37-44.

36. Therapeutic use exemptions (internet). Available from http://www.uci.ch/ clean-sport/therapeutic-use-exemptions. [Accessed 6 August 2015].

37. Levine WN, Bergfeld JA, Tessendorf W, Moorman CT. $3^{\text {rd }}$ Intramuscular corticosteroid injection for hamstring injuries: a 13-year experience in the National Football League. Am J Sports Med 2000 May-Jun;28(3):297-300.

38. Gunter $P$, Schwellnus $M$, Fuller P. Local corticosteroid injection in iliotibial band friction syndrome in runners: a randomised controlled trial Br J Sports Med 2004 Jun;38(3):269-272.

39. Haralambous CP, Tryfonidis $M$, Sadiq $S$, et al. Septic arthritis following intra-articular steroid injection of the knee-a survey of current practice regarding antiseptic technique used during intra-articular steroid injection of the knee. Clin Rheumatol 2003;22:386-90.

40. Fadale PD, Wiggins ME. Corticosteroid injections: their use and abuse. J Am Acad Orthop Surg 1994;2:133-140.

41. Nepple JJ, Matava MJ. Soft Tissue Injections in the Athlete. Sports Health 2009 Sep;1(5):396-404.

42. Fredberg U. Local corticosteroid injection in sport: review of literature and guidelines for treatment, Sacand J Med Sci Sports 1997;7:131-139.

43. Dvorak J, Feddermann N, Grimm K. Glucocorticosteroids in football: use and misuse Br J Sports Med 2006;40(Suppl I):i48-i54.

44. Richie CA, Briner WW. Corticosteroid injection for the treatment of de Quervain's tenosynovitis: a pooled quantitative literature evaluation. J Am Board Fam Pract 2003;16(2):102-106.

45. Murphy D, Failla JM, Koniuch MP. Steroid versus placebo injection for trigger finger. J Hand Surg 1995;20A:628-631. 